\title{
TU6A-1
}

\section{Hilbert fractal curves for HTS miniaturized filters}

\author{
M. Barra ${ }^{1}$, C. Collado ${ }^{2}$, J. Mateu, J.M. O'Callaghan ${ }^{2}$ \\ ${ }^{1}$ INFM-Dipartimento di Scienze Fisiche, Università dei Napoli “Federico II", Napoli, Italy \\ ${ }^{2}$ UPC (Universitat Politècnica de Catalunya), Campus Nord, Barcelona, Spain \\ ${ }^{3}$ CTTC (Centre Tecnològic de Telecomunicacions de Catalunya), Barcelona, Spain
}

\begin{abstract}
This work presents novel compact High Temperature Superconductor (HTS) microstrip resonators based on Hilbert fractal curves. Their miniaturization performance has been investigated, emphasizing the parameters which allow to obtain a good trade off between compact size and quality factor. Hilbert resonators with different arrangements have been designed and assessed by using full wave simulators. In oder to experimentally verify our analysis, a four pole quasi elliptic filter, in the cascade quadruplet configuration, with $f_{0}$ close to $2.45 \mathrm{GHz}$, have been fabricated by patterning a $10 \times 10 \mathrm{~mm}^{2}$ YBCO film on $\mathrm{MgO}$ substrates. Minimum insertion losses of the order of $(0.1-0.2) \mathrm{dB}$ have been measured.
\end{abstract}

Index Terms - Microstrip filters, High-Temperature Superconductors, Fractals, Microwave devices, Mobile Communication.

\section{INTRODUCTION}

In recent years, the miniaturization trend of planar filters has received a new and growing interest because of the discovery of HTS. The miniaturization causes the corresponding increase of current density in the strip. High current densities in conventional metallic strips give rise to a very large amount of dissipative losses with a dramatic and unacceptable worsening of filter performance. In contrast, the very low surface resistance of HTS films at microwave frequencies allows to fabricate high performance compact planar resonators and filters.

Nowadays, HTS filters seem to be me of the most adequated to satisfy the needs of the modern telecommunication systems, when maximum compactness of the microwave circuitry and still stringent filter performance are required [1]. In this context, new highly compact HTS resonators have been recently proposed and successfully tested [2]-[5]. Despite their different shapes, all these structures are based on the miniaturizing principle to fold the elementary straight line resonator in very sophisticated ways in order to fit it in a compact area. Considering this scenario, in the present paper we report the results, in terms of miniaturization and overall performance, obtained by using HTS filters shaped as Hilbert curves [6].

\section{HILBERT MICROSTRIP RESONATORS}

In 1892, in a study about the existence of special curves which present space filling capabilities and the property of being everywhere continuous, the German mathematician David Hilbert presented the sets of curves shown in Fig.1 for the first four iterations $(k=1 . .4)$. As evidenced by the presence of the background grid, the Hilbert curve with $k=1$ connects the centres of the four parts in which is divided the original square. For $k=2$, the same criterion can be applied dividing the square in 16 parts and connecting the centres in the same way. For the general $\mathrm{k}^{\text {th }}$ iteration, $2^{2 \mathrm{k}}$ divisions are realized and consequently the curve will be composed of $\left(2^{2 \mathrm{k}}-1\right)$ segments, all with the same length.

Recently and accordingly to what just mentioned, the miniaturization performances by means of Hilbert curves in the fabrication of small antennas have been intensively investigated [7]-[9]. These studies have clearly shown that, increasing the iteration level while keeping fixed the external side ( $S$, see Fig. 1 ), the resonant frequencies of a Hilbert antenna lower whereas the radiation characteristics worsen by decreasing the radiation resistance. From Hilbert microstrip resonators point of view, this last property suggests their good performances in lowering the packaging losses due to the radiated field. Moreover, analyzing the data reported in literature, it can be observed that in every $k$ case, the fundamental resonance frequency is however higher than the fundamental frequency of a $N / 4$ monopole with the same length. This phenomenon is due to the couplings between the different turns of the Hilbert resonator which practically define an equivalent shorter path for the current. Obviously this effect gets stronger with $k$ increasing, since a reduction of the interspacing between turns $(g$ ) takes place. Thus, a saturation the structure miniaturization capability occurs for large values of $k$. In other words, the ratio $f(\mathrm{k}+1) / \mathrm{f}_{0}(\mathrm{k})$ between the 
resonant frequencies of two consecutive iterations tends rapidly towards 1 , instead of 0.5 , which should be, considering the almost doubling of the length. This means, from a practical point of view, that only the first iterations ( $k \leq 5$ or 6) of a Hilbert resonator guarantee an effective miniaturization improvement.

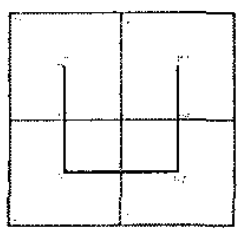

$k=1$

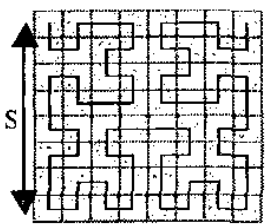

$k=3$

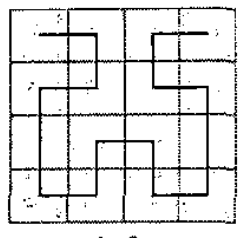

$\mathrm{k}=2$

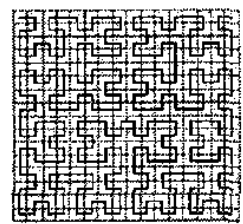

$k=4$
Fig. 1. Hilbert curves with different iteration levels $\mathbf{k}$.

In Hilbert microstrip resonators, the strip width $w$ and the interspacing between turns $g$ are the parameters which define the trade off between miniaturization and quality factor $Q$. To obtain highly miniaturized resonators, which in our case means adopting curves with high $k$, the value of $w$ is considerably narrowed. In turn, the current density increase, the dissipation losses increase and the quality factor decrease.

To illustrate this, Fig.2a shows some simulated results on how the resonant frequency $f_{0}$, depends on the external side $S$, as a function of Hilbert curve iteration $k$. In this simulation we have kept the ratio width of the strip and the interspacing between turns constant to 1 (i.e. $w / g=1$ ). On the other hand, Fig. $2 \mathrm{~b}$ reports the required $w$ to fit a $k$ Hilbert curve inside a square $\mathrm{SxS}$, while keeping $w / g=1$.

From this preliminary analysis, the Hilbert resonator with $k=4$ and $w / g=1$ shown in Fig. 3 seems to be the best candidate to assure a good trade-off between miniaturization level and $w$ at $f_{0}=2 \mathrm{GHz}$. In this case, the strip width is $115 \mu \mathrm{m}$ and the external dimension $S$ is only $3.58 \mathrm{~mm}$ (which corresponds to $0.06 \lambda$, being $\lambda$ the wavelength for a $50 \Omega$ transmission line on $\mathrm{MgO}$ ).

The $k=4$ Hilbert resonator was realized on a $10 \times 10 \mathrm{~mm}^{2}$ double sided $700 \mathrm{~nm}$ YBCO thin film on $\mathrm{MgO}$. The resonator was measured at $\mathrm{T}=77 \mathrm{~K}$ in a liquid nitrogen bath resulting a $Q$ of about 30000 . It is worth to mention that this value is in very good agreement with the value predicted by Momentum software [10]. Moreover, it is very similar to those reported in very recent papers for other compact resonators [4] and [5].
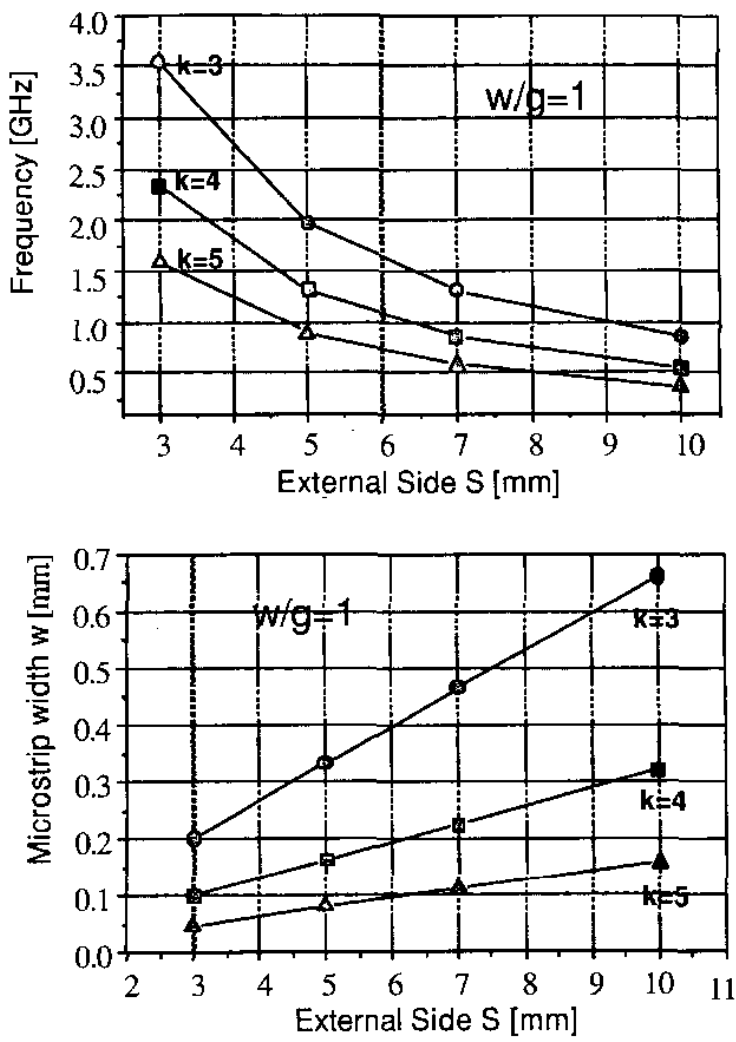

Fig. 2. a) fundamental resonant frequencies of hilbert resonators with $k=3,4,5$ and b) corresponding microstrip widths as a function of the external side $s(\mathrm{k})$.

\section{FILTER DESIGNS AND EXPERIMENTAL RESULTS}

In this section we report the design and test of a four pole quasielliptic filter based on the $k=4$ Hilbert resonator (Fig.3). To do so, we slightly modify the layout of the resonator to fulfill the requirements to realize quasielliptic filters.

Quasi-elliptic filters need out-of-phase coupling between non-adjacent resonators [3]. In order to achieve out-ofphase couplings, the Hilbert resonator has been provided with a capacitive load by lengthening the ends of the resonator. The outline of the resonator is shown in Fig. 4a.

Following the procedure described in [11], a four pole quasi-elliptic filter can be implemented with a conventional cascaded-quadruplet topology. The couplings between the resonators are obtained by properly fixing the spacing 
between them. Input and output port (feed lines) are coupled to the first and last resonator, respectively.

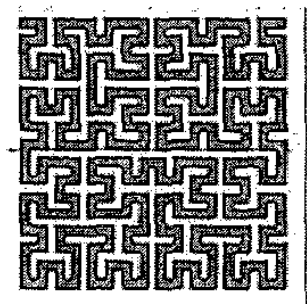

Fig. 3. Hilbert resonator with $\mathrm{k}=4$.

According to our results, with this resonator it is not possible to obtain the desired values of Qext by coupling with capacitive gaps, or by direct connection to the strip (tapped line configuration) [11]. Capacitive coupling provides values of Qext larger than 200, suitable for filters with fractional bandwidths lower than $0.5 \%$, whereas direct conection of the feedline with the resonator strip results in values of Qext lower than 20 (adequate for fractional bandwidths larger than $3 \%$, i.e. $60 \mathrm{MHz}$ at $2 \mathrm{GHz}$ ). This problem could be solved if the feedline could be connected closer to the point in the resonatro strip where the current is maximum. To achieve this, we have rearranged the Hilbert resonator as depicted in Fig. 4b. In this new configuration, the feed lines can be conected close to the current maximum, and Qext is determined by the separation between the contact point and the point where current is maximum.

Notice that the rearranging of Fig. $4 \mathrm{~b}$ consists on changing the orientation of two of the four component elements of $k=3$, in order to provide a double axial symmetric structure which makes accessible the point of maximum current.

Finally, using Fig. $4 \mathrm{~b}$ structure a quasielliptic filter with $20 \mathrm{MHz}$ bandwidth centred at $2.45 \mathrm{GHz}$ has been designed. The filter configuration is shown in Fig.5. The filter has been fabricated using a $10 \times 10 \mathrm{~mm}^{2}$ double sided $700 \mathrm{~nm}$ YBCO thin film deposited on $0.508 \mu \mathrm{m}$ thick $\mathrm{MgO}$ substrate. The basic resonator dimensions are $2.68 \mathrm{~mm} \times 2.28 \mathrm{~mm}$ with a microstrip width of $90 \mu \mathrm{m}$. The overall dimensions of the filter are $7.9 \mathrm{~mm} \times 6.2 \mathrm{~mm}$. The filter has been measured in a liquid nitrogen bath at $P_{i n}=0 \mathrm{dBm}$. A slight shift in the central frequency has been observed between the simulated $\left(f_{0}=2.45 \mathrm{GHz}\right)$ and the measured $\left.f_{i}=2.438 \mathrm{GHz}\right)$ responses. Fig. 6 compares the measured response without tuning- with a shifted version of the simulated response. In Fig.6a, we see the agreement in bandwidth and transmission zero position between measured (continuous line) and simulated (dashed line) response. a)

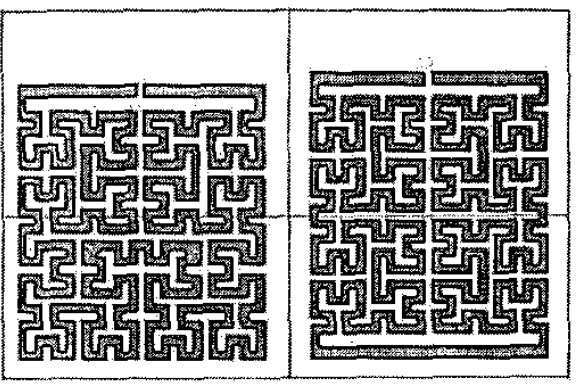

b)

Fig. 4. a) Hilbert resonator with capacitive load b) Hilbert resonator arrangement with accessible point of maximum current.

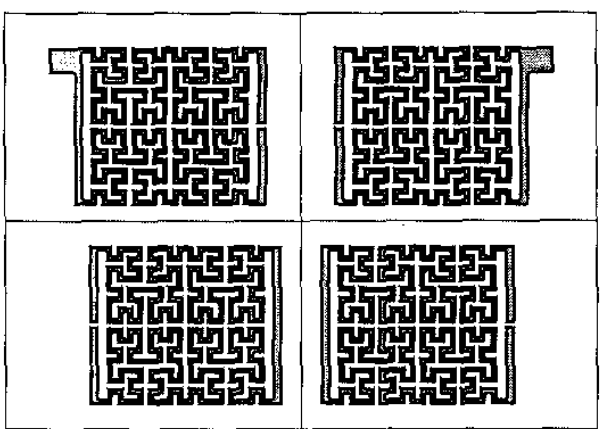

Fig. 5. Quasi-elliptic four pole filter.

The measured $3 \mathrm{~dB}$ bandwidth (Fig.6b) differs $1 \mathrm{MHz}$ of the one expected from simulations. Fig. $6 \mathrm{~b}$ also shows a $0.2 \mathrm{~dB}$ minimum insertion losses and a ripple distortion of $0.6 \mathrm{~dB}$, which corresponds to the $8 \mathrm{~dB}$ measured return losses. These discrepances between measured and simulated in-band response might be attributed to detunning which, in turn, reduces the steepness at the upper bound of the passband and worsens the symmetry of the frequency response. The detunning may come from many parasitic effects, such as unwanted coupling, deviation in thickness substrate, tolerance in the fabrication process and so on [11].

As a last point, it is worthwhile to mention the absence of the second harmonic peak $2 \mathrm{f}_{0}$ as a consequence of the capacitive load of each resonator. In practice, at $2 \mathrm{f}_{0}$, the length of the resonators are as long as one wavelength, thus their ends present charges with the same sign (i.e. all couplings are in-phase) reducing considerably the effects of the equivalent capacitance [12]. The data shown in Fig. $6 \mathrm{c}$ confirm the absence of the second harmonic. 


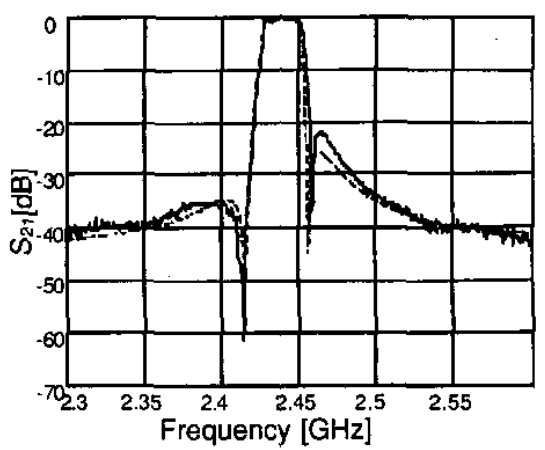

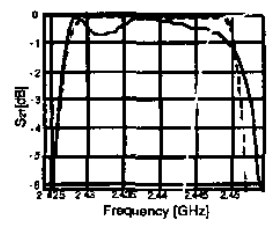

b)

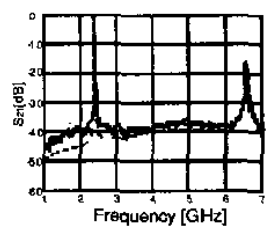

c)
Fig. 6. a) Comparison between measured (continuous line) and simulation (dashed line) filter responses. In band (b) and large range (c) responses.

\section{CONCLUSION}

In this work the miniaturization performance of a novel type of HTS microstrip resonator based on the Hilbert curves has been investigated. The fractal structure was analyzed considering different levels of iteration and putting in evidence the incidence of the different geometric parameters on the obtainable miniaturization level. The performance in terms of quality factor of a single Hilbert resonator with a side of $3.58 \mathrm{~mm}$ and $\mathrm{f}_{\mathrm{h}}=2 \mathrm{GHz}$, has been measured at $77 \mathrm{~K}$ showing a $Q$ of about 30000 . By using a commercial $10 \times 10 \mathrm{~mm}^{2} \mathrm{YBCO}$ thin film on $\mathrm{MgO}$, a four pole quasi-elliptic filter centred close to $2.45 \mathrm{GHz}$ has been fabricated, showing the flexibility of this type of resonator. Agreement between between measurements and simulations has been observed. The measured insertion losses (0.1-0.2) dB confirm the good trade off between high performance and reduced dimensions.

\section{ACKNOWLEDGEMENT}

The authors wish to thank the extensive technical work provided by L. Enrique in the fabrication and preparation of all the tested devices. We also thank J. Romeu and J. $\mathrm{M}^{\mathrm{a}}$ González and A. Cassinese for the stimulating discussions. This work is supported by the Spanish Ministry of Science and Technology through project MAT2002-04551-C03-03 and by Generalitat de Catalunya (DURSI) through grant 2001SGR 0026.

\section{REFERENCES}

[1] M.J. Lancaster, Microwave passive device application of high temperature superconductors, Cambrige University Press, 1997.

[2] M. Reppel and J.C. Mage "Superconducting microstrip bandpass filter on $\mathrm{LaAlO}_{3}$ with high out-of-Band rejection", IEEE Microwave and Guided letters, vol.10 no.5, pp. 180182, May 2000.

[3] J. S. Hong, M.J. Lancaster, D. Jedamzik, R. B, Greed and J. C. Mage "On the performance of HTS nicrostip quasi elliptic function filters for mobile communications application", IEEE Trans On Microwave Theory and Techniques, vol.48, no.7, pp.1240-1246, July 2000.

[4] F. Huang "Ultra-compact superconducting narrow band filters using single and twin spiral resonators", IEEE Trans On Microwave Theory and Techniques, vol. 51, no.2, pp. 487-491, Feb.2003.

[5] G.L. Matthaei "Narrow band, fixed tuned and tunable bandpass filters with Zig-Zag hairpin comb resonators" IEEE Trans. On Microwave Theory and Techniques, vol.51, no.4, pp. 1214-1219, April 2003.

[6] J. M. O'Callaghan, C. Puente, N. Duffo, C. Collado, E. Rozan. Fractal and Space-Filling Transmission Lines, Resonators, Filters and Passive Network Elements PatentWO0154221. (Fractus S.A.)

[7] J. Anguera, C. Puente, E. Martinez and E. Rozan, "The fractal Hilbert monopole: A two-dimensional wire" Microwave and Optical technology letters, vol.36, no.2, pp. 102-104, January 2003.

[8] S.R. Best, "A comparison of the performance properties of the Hilbert curve fractal and meander line monopole antennas" Microwave and Optical technology letters, vol.35, no.4, pp. 258-262, November 2002.

[9] J.M. Gonzalez-Arbesú, S. Blanch, J. Romeu, "The Hilbert curve as a small self-resonant monopole from a practical point of view", Microwave an Optical Technology Letters, vol. 39, no. 1, pp.45-49, October 2003.

[10] Momentum, Adranced Design System (ADS), Agilent Technologies.

[11] J. S. Hong and, M.J. Lancaster Microstrip filters for RF/microwave applications, John Wiley \& Sons, 2001.

[12] J. Zhou, M. J. Lancaster and F. Huang, "Superconducting microstrip filters using compact resonators with doublespiral inductors and interdigital capacitors", 2003 IEEE MTTSymp International, pp.1889-1892. 